\title{
On the Regularity for Solutions of the Micropolar Fluid Equations
}

\author{
Elva Ortega-Torres (*) - Marko Rojas-Medar (**)
}

ABstRact - We give sufficient conditions on the kinematics pressure in order to obtain regularity and uniqueness of the weak solutions to the micropolar fluid equations.

\section{Introduction.}

We consider the regularity of the weak solutions for the equations that describes the motion of a viscous incompressible micropolar fluids in a bounded domain $\Omega \subset \mathbb{R}^{3}$, with smooth boundary $\partial \Omega$ over a time interval $[0, T], 0<T<+\infty$. These equations are given by

$$
\frac{\partial \boldsymbol{u}}{\partial t}+\boldsymbol{u} \cdot \nabla \boldsymbol{u}-v_{1} \Delta \boldsymbol{u}+\nabla p=2 \mu_{r} \operatorname{rot} \boldsymbol{w}+\boldsymbol{f},
$$

(2) $\quad \operatorname{div} \boldsymbol{u}=0$

$$
\frac{\partial \boldsymbol{w}}{\partial t}+\boldsymbol{u} \cdot \nabla \boldsymbol{w}-v_{2} \Delta \boldsymbol{w}-v_{3} \nabla \operatorname{div} \boldsymbol{w}+4 \mu_{r} \boldsymbol{w}=2 \mu_{r} \operatorname{rot} \boldsymbol{u}+\boldsymbol{g},
$$

where $v_{1}=\mu+\mu_{r}, v_{2}=c_{a}+c_{d}, v_{3}=c_{0}+c_{d}-c_{a}$.

The unknowns are the functions $\boldsymbol{u}(x, t) \in \mathbb{R}^{3}, \boldsymbol{w}(x, t) \in \mathbb{R}^{3}$ and $p(x, t) \in \mathbb{R}$ which denote the linear velocity vector, the angular velocity vector of rotation of particles, and the pressure of the fluid, respectively. The functions $\boldsymbol{f}(x, t) \in \mathbb{R}^{3}$ and $\boldsymbol{g}(x, t) \in \mathbb{R}^{3}$ are given, and denote external

(*) Indirizzo dell'A.: Departamento de Matemáticas, Universidad Católica del Norte, Av. Angamos 0610, Casilla 1280, Antofagasta, Chile.

E-mail: eortega@ucn.cl

(**) Indirizzo dell'A.: Depto. de Ciencias Básicas, Univ. del Bio-Bio, Campus Fernando May, Casilla 447, Chillán, Chile.

E-mail: marko@ueubiobio.cl 
sources of linear and angular momentum, respectively. The positive constants $\mu, \mu_{r}, c_{0}, c_{a}$ and $c_{d}$, characterize isotropic properties of the fluid: $\mu$ is the usual Newtonian viscosity; $\mu_{r}, c_{0}, c_{a}, c_{d}$ are new viscosities related to the appearance of the field of internal rotation $\boldsymbol{w}$ and satisfy $c_{0}+c_{d}>c_{a}$.

Together with the equations (1)-(3) we prescribe the following boundary and initial conditions

$$
\begin{array}{llll}
\boldsymbol{u}(x, t)=0, & \boldsymbol{w}(x, t)=0 & \text { on } & S_{T} \\
\boldsymbol{u}(x, 0)=\boldsymbol{u}_{0}(x), & \boldsymbol{w}(x, 0)=\boldsymbol{w}_{0}(x) & \text { in } & \Omega .
\end{array}
$$

For the derivation and physical discussion of the equations (1)-(3) see [7] and [14]. The existence of diverse notions of solutions for the system (1)-(3) has been much studied, as in [13], [17], [18] and [23]. We observe that the equations (1)-(3) include as a particular case the classical Navier-Stokes equations, which widely has been studied (see, for instance, the classical books [12], [21] and the references there in).

In the theory of the three-dimensional Navier-Stokes equations, the problems of uniqueness of weak solutions is the most difficult ones (see for instance [21], p. 297, [12]). For sufficiently smooth solutions, the uniqueness is proved quite easily, while in the class of weak solutions the problem of uniqueness (or non-uniqueness) remain open to discussion. There exist many works devoted to these questions. A pioneer work in this direction was give by Serrin [19], Serrin proved that a weak solution $(\boldsymbol{u}, p)$ is regular if the velocity $\boldsymbol{u}$ satisfies some suitable extra conditions. After many authors extend the result of Serrin, see for instance [11], [22], [8], [1], [17].

The pressure term is source of problems for understanding of the Navier-Stokes equations, since it plays a role seemed to a Lagrange multiplier to enforce the incompressibility constraint. Thus, an other interesting question is to look for conditions involving the pressure and not the velocity, as it has been done in almost all the literature. A first sufficient condition for regularity involving the pressure alone, was given by Kaniel in [10] who proved that $p \in L^{\infty}\left(0, T ; L^{q}(\Omega)\right)$, for $q>12 / 5$ is a sufficient condition for the smoothness of weak solutions of the three-dimensional Navier-Stokes equations. This approach was ignored by many years but, recently, many authors have write extensions of the results by Kaniel [10], see for instance [2], [3], [16], [5], [6]. Following the idea of Kaniel it is well known that if $\boldsymbol{u}_{0} \in V$, there exists an unique strong local solution for the Navier-Stokes equations, and if $\left\|\boldsymbol{u}_{0}\right\|_{V}$ is small enough and the external force is zero, the solution is global. Since the weak global solution also exist, then the local strong solution is 
equal to the weak solution on the maximal interval of existence, say $\left[0, T^{*}\right)$, with $0<T^{*}<T$. Thus, the following question comes to our mind: what conditions on the pressure implies that the unique local strong solution is in fact global?, in other words, it is possible to change the condition on $\left\|\boldsymbol{u}_{0}\right\|_{V}$ by another that involve only the pressure?. If we have an affirmative answer, we would have the uniqueness of weak solutions. It is important to say that these results is not directly comparable to the Serrin, since the initial condition is take in different spaces. Moreover, it will be interesting to know if it is possible change the hypotheses of Serrin by another that involve only the pressure, but this is a difficult question. In fact, in an early work Heywwod and Walsh [9] give an interesting result on the behavior of the pressure in $t=0$ when the initial condition $\boldsymbol{u}_{0} \in V$. They show that there exist initial conditions $\boldsymbol{u}_{0} \in V$ such that limsup $\|p\|_{L^{2}(\Omega)}=+\infty$, which implies that $\limsup \|p\|_{L^{q}(\Omega)}=+\infty$ for any $q \geq 2$.

By the other hand, the condition given by Kaniel is: $\|p\|_{L^{q}(\Omega)} \leq C_{0}$ for each $t$ in the interval of existence of the weak solution, with $q>\frac{12}{5}$, in particular, this implies that the $\limsup _{t \rightarrow 0^{+}}\|p\|_{L^{2}(\Omega)}$ is finite and consequently some degree of regularity on the pressure is in fact necessary. Thus, other interesting question in this direction is find the minimal hypotheses on the pressure. The work of Berselli [5] is in that direction. In fact, the Berselli's results, roughly speaking, are the $L^{q}$ - versions of the Kaniel result $(q>3)$.

The purpose of this paper is to extend the proof presented in [5] to the micropolar equations, and thus, we research the same level of knowledge as the one in the case of the classical Navier-Stokes equations. We note that, as remarked in [5], the proof is done by following techniques introduced in [4] and developed in [3].

An outline of this paper is as follows. In Section 2, we introduce some notations and functions spaces, and we state our main theorem. In Section 3 , we prove some estimates for the proof of our main result. Finally, in Section 4 , we prove our main theorem.

\section{Statements, notations and main result.}

We denote by $L^{p}(\Omega), 1 \leq p \leq+\infty$ the usual Lebesgue spaces of scalar functions and for vector value functions we define $\boldsymbol{L}^{p}(\Omega)=$ $=\left(L^{p}(\Omega)\right)^{3}$ with norm $\|\boldsymbol{v}\|_{p}=\||\boldsymbol{v}|\|_{p}$ where $|\cdot|$ denotes the Euclidean 
length. As customary, $\boldsymbol{H}_{0}^{1}(\Omega)$ denotes the Sobolev space given by the functions vanishing on the boundary and belonging to $\boldsymbol{L}^{2}(\Omega)$ with their first-order distributional derivatives, and the norm is defined by $\|\boldsymbol{v}\|_{H_{0}^{1}} \equiv\||\nabla \boldsymbol{v}|\|_{2}$. When $\boldsymbol{X}$ is a Banach space, we denote by $C(0, T ; \boldsymbol{X})$ the set of continuous functions on [0,T] with values in $\boldsymbol{X}$ and by $L^{p}(0, T ; \boldsymbol{X})$ the Banach space of measurable functions $\boldsymbol{v}$ with values in $\boldsymbol{X}$, endowed with the norm

$$
\|\boldsymbol{v}\|_{L^{p}(0, T ; X)}=\left(\int_{0}^{T}\|\boldsymbol{v}(t)\|_{X}^{p} d t\right)^{1 / p}
$$

Let $\boldsymbol{C}_{0}^{\infty}(\Omega)$ denote the set of all $C^{\infty}$-real vector functions $\boldsymbol{v}$ with compact support in $\Omega$, and $\boldsymbol{C}_{0, \sigma}^{\infty}(\Omega)$ denotes the functions $\boldsymbol{v} \in \boldsymbol{C}_{0}^{\infty}(\Omega)$ such that $\operatorname{div} \boldsymbol{v}=0$. Then, we introduce the following function spaces

$$
\begin{aligned}
H_{\alpha} & =\text { closure of } \boldsymbol{C}_{0, \sigma}^{\infty}(\Omega) \text { in } \boldsymbol{L}^{\alpha}(\Omega), \\
G_{\alpha} & =\text { closure of } \boldsymbol{C}_{0}^{\infty}(\Omega) \text { in } \boldsymbol{L}^{\alpha}(\Omega), \\
\boldsymbol{V} & =\text { closure of } \boldsymbol{C}_{0, \sigma}^{\infty}(\Omega) \text { in } \boldsymbol{H}_{0}^{1}(\Omega) .
\end{aligned}
$$

We denote by the same $C$ various positive constants and by $C(\cdot, \cdot, \ldots)$ the constants depending only on the quantities appearing in parentheses.

Also, we denote the classical bilinear and trilinear forms by

$$
(\nabla \boldsymbol{u}, \nabla \boldsymbol{v})=\sum_{i, j=1}^{3} \int_{\Omega} \frac{\partial u_{j}}{\partial x_{i}} \frac{\partial v_{j}}{\partial x_{i}} d x, \quad(\boldsymbol{u} \cdot \nabla \boldsymbol{v}, \boldsymbol{w})=\sum_{i, j=1}^{3} \int_{\Omega} u_{j} \frac{\partial v_{i}}{\partial x_{j}} w_{i} d x
$$

for all $\boldsymbol{u}, \boldsymbol{v}, \boldsymbol{w}$ where the integrals make sense. Moreover, the trilinear form has the properties

$$
\left(\boldsymbol{u} \cdot \nabla \boldsymbol{v},|\boldsymbol{v}|^{\alpha-2} \boldsymbol{v}\right)=0, \quad \forall \boldsymbol{u} \in \boldsymbol{V}, \forall \boldsymbol{v} \in \boldsymbol{H}_{0}^{1}(\Omega) .
$$

For simplicity of notations, we consider the following quantities (introduced in reference [4])

$$
N_{\alpha}^{\alpha}(\boldsymbol{u})=\left\||\boldsymbol{u}|^{\frac{\alpha}{2}-1}|\nabla \boldsymbol{u}|\right\|_{2}^{2}, \quad M_{\alpha}^{\alpha}(\boldsymbol{u})=\left\|\left.|\nabla| \boldsymbol{u}\right|^{\frac{\alpha}{2}} \mid\right\|_{2}^{2}
$$

and, in the calculations will be used the inequalities

$$
\begin{aligned}
& \|\boldsymbol{u}\|_{3 \alpha}^{\alpha} \leq C_{0} M_{\alpha}^{\alpha}(\boldsymbol{u}), \quad \forall|\boldsymbol{u}|^{\frac{\alpha}{2}} \in \boldsymbol{H}_{0}^{1}(\Omega), \\
& |\nabla| \boldsymbol{v} \| \leq|\nabla \boldsymbol{v}|, \quad \forall \boldsymbol{u} \in \boldsymbol{H}_{0}^{1}(\Omega),
\end{aligned}
$$


and for all $\boldsymbol{v} \in \boldsymbol{H}_{0}^{1}(\Omega)$, the following equalities

$$
\begin{aligned}
(\operatorname{rot} \boldsymbol{v}, \boldsymbol{w}) & =(\boldsymbol{v}, \operatorname{rot} \boldsymbol{w}), \quad \forall \boldsymbol{w} \in \boldsymbol{H}_{0}^{1}(\Omega), \\
\operatorname{rot}\left(|\boldsymbol{v}|^{\alpha-2} \boldsymbol{v}\right) & =|\boldsymbol{v}|^{\alpha-2} \operatorname{rot} \boldsymbol{v}+(\alpha-2)|\boldsymbol{v}|^{\alpha-3} \nabla|\boldsymbol{v}| \times \boldsymbol{v}, \\
\operatorname{div}\left(|\boldsymbol{v}|^{\alpha-2} \boldsymbol{v}\right) & =|\boldsymbol{v}|^{\alpha-2} \operatorname{div} \boldsymbol{v}+(\alpha-2)|\boldsymbol{v}|^{\alpha-3} \boldsymbol{v} \cdot \nabla|\boldsymbol{v}|, \\
\left\||\boldsymbol{v}|^{\alpha-2} \boldsymbol{v}\right\|_{\frac{\alpha}{\alpha-1}} & =\|\mid \boldsymbol{v}\|_{\alpha}^{\alpha-1} .
\end{aligned}
$$

Now, we set out our main result

THEOREM 2.1. Let $(\boldsymbol{u}, \boldsymbol{w})$ be a weak solution of the problem (1)-(5) with initial conditions $\boldsymbol{u}_{0} \in H_{\alpha}, \boldsymbol{w}_{0} \in G_{\alpha}$ and $\boldsymbol{f}, \boldsymbol{g} \in L^{\alpha}\left(0, T ; L^{\alpha}(\Omega)\right)$ for $\alpha>3$. Ifp the associated pressure satisfies

$$
p \in L^{\alpha}\left(0, T ; L^{\frac{3 x}{\alpha+1}}(\Omega)\right)
$$

then the weak solution satisfies

$$
(\boldsymbol{u}, \boldsymbol{w}) \in C\left(0, T ; H_{\alpha}\right) \times C\left(0, T ; G_{\alpha}\right)
$$

and

$$
\left(|\boldsymbol{u}|^{\frac{\alpha}{2}},|\boldsymbol{w}|^{\frac{\alpha}{2}}\right) \in L^{2}\left(0, T ; \boldsymbol{H}_{0}^{1}(\Omega)\right) \times L^{2}\left(0, T ; \boldsymbol{H}_{0}^{1}(\Omega)\right) .
$$

REMARK 2.2. To the Navier-Stokes equations it is well known that a weak solution in the sense of Leray-Hopf (see [19], [15], [20], [21]) is regular if

$$
\boldsymbol{u} \in L^{r}\left(0, T ; \boldsymbol{L}^{s}(\Omega)\right), \quad \frac{2}{r}+\frac{3}{s} \leq 1, \quad s>3 .
$$

REMARK 2.3. If we put $r=\alpha$ and $s=3 \alpha /(\alpha+1)$ we get as a sufficient condition for regularity that

$$
\text { (18) } p \in L^{r}\left(0, T, L^{s}(\Omega)\right) \quad \text { with } \quad s>9 / 4 \quad \text { and } \quad \frac{2}{r}+\frac{3}{s}=1+\frac{3}{r} \text {. }
$$

Note that the right-hand side in the equality of (18) is a number greater than 1 and strictly lower than 2 . Note that if we could take $r=3$ we would get a pressure belonging to $L^{3}\left(0, T ; L^{\frac{9}{4}}(\Omega)\right)$. By taking into account that $p \simeq|\boldsymbol{u}|^{2}$, this condition is equivalent to $\boldsymbol{u} \in L^{6}\left(0, T ; \boldsymbol{L}^{\frac{9}{2}}(\Omega)\right)$, which satisfies (17).

\section{A priori estimates.}

With the notations given in Section 2 and in order to prove the main Theorem 2.1, we give the following Lemma: 
LEMMA 3.1. Let $(\boldsymbol{u}, p, \boldsymbol{w})$ be a smooth solution of the micropolar equations (1)-(5) in $\Omega \times(0, T]$. Then the following estimate is satisfied

$\frac{1}{\alpha} \frac{d}{d t}\left(\|\boldsymbol{u}\|_{\alpha}^{\alpha}+\|\boldsymbol{w}\|_{\alpha}^{\alpha}\right)+\frac{3 v_{1}}{4} N_{\alpha}^{\alpha}(\boldsymbol{u})+\frac{v_{2}}{2} N_{\alpha}^{\alpha}(\boldsymbol{w})+4 v_{1} \frac{(\alpha-2)}{\alpha^{2}} M_{\alpha}^{\alpha}(\boldsymbol{u})+C_{1} \frac{(\alpha-2)}{\alpha^{2}} M_{\alpha}^{\alpha}(\boldsymbol{w})$

$$
+4 \mu_{r}\|\boldsymbol{w}\|_{\alpha}^{\alpha} \leq-\left(\nabla p,|\boldsymbol{u}|^{\alpha-2} \boldsymbol{u}\right)+C\left(\|\boldsymbol{f}\|_{\alpha}^{\alpha}+\|\boldsymbol{g}\|_{\alpha}^{\alpha}\right)+C\left(\|\boldsymbol{u}\|_{\alpha}^{\alpha}+\|\boldsymbol{w}\|_{\alpha}^{\alpha}\right)
$$

where $C_{1}=4 v_{2}-\alpha v_{3}>0$.

Proof. We multiply both sides of (1) by $|\boldsymbol{u}|^{\alpha-2} \boldsymbol{u}$ (as in the proof of Lemma 1.1, [4]) and then doing integration by parts in $\Omega$, taking into account (6) and (10), it follows that

$$
\begin{aligned}
& \frac{1}{\alpha} \frac{d}{d t}\|\boldsymbol{u}\|_{\alpha}^{\alpha}+v_{1} N_{\alpha}^{\alpha}(\boldsymbol{u})+4 v_{1} \frac{(\alpha-2)}{\alpha^{2}} M_{\alpha}^{\alpha}(\boldsymbol{u}) \\
& \quad \leq-\left(\nabla p,|\boldsymbol{u}|^{\alpha-2} \boldsymbol{u}\right)+2 \mu_{r}\left(\boldsymbol{w}, \operatorname{rot}\left(|\boldsymbol{u}|^{\alpha-2} \boldsymbol{u}\right)\right)+\left(\boldsymbol{f},|\boldsymbol{u}|^{\alpha-2} \boldsymbol{u}\right) .
\end{aligned}
$$

In analogous form multiplying (3) by $|\boldsymbol{w}|^{\alpha-2} \boldsymbol{w}$, taking into account (6), (10) and (12), we get

$$
\begin{aligned}
& \frac{1}{\alpha} \frac{d}{d t}\|\boldsymbol{w}\|_{\alpha}^{\alpha}+v_{2} N_{\alpha}^{\alpha}(\boldsymbol{w})+4 v_{2} \frac{(\alpha-2)}{\alpha^{2}} M_{\alpha}^{\alpha}(\boldsymbol{w})+v_{3}\left\||\boldsymbol{w}|^{\frac{\alpha}{2}-1}|\operatorname{div} \boldsymbol{w}|\right\|_{2}^{2}+4 \mu_{r}\|\boldsymbol{w}\|_{\alpha}^{\alpha} \\
& \quad \leq 2 \mu_{r}\left(\boldsymbol{u}, \operatorname{rot}\left(|\boldsymbol{w}|^{\alpha-2} \boldsymbol{w}\right)\right)+\left(\boldsymbol{g},|\boldsymbol{w}|^{\alpha-2} \boldsymbol{w}\right)+v_{3}(\alpha-2)\left(\operatorname{div} \boldsymbol{w},|\boldsymbol{w}|^{\alpha-3} \boldsymbol{w} \nabla|\boldsymbol{w}|\right) .
\end{aligned}
$$

Now, taking into account (11) and (9), we have

$$
\begin{aligned}
\left(\boldsymbol{w}, \operatorname{rot}\left(|\boldsymbol{u}|^{\alpha-2} \boldsymbol{u}\right)\right) & =\left(\boldsymbol{w},|\boldsymbol{u}|^{\alpha-2} \operatorname{rot} \boldsymbol{u}\right)+(\alpha-2)\left(\boldsymbol{w},|\boldsymbol{u}|^{\alpha-3} \nabla|\boldsymbol{u}| \times \boldsymbol{u}\right) \\
& =\left(|\boldsymbol{w}|,|\boldsymbol{u}|^{\alpha-2}|\nabla \boldsymbol{u}|\right)+(\alpha-2)\left(|\boldsymbol{w}|,|\boldsymbol{u}|^{\alpha-3}|\nabla| \boldsymbol{u}|\times \boldsymbol{u}|\right) \\
& \leq\left(|\boldsymbol{w}|,|\boldsymbol{u}|^{\alpha-2}|\nabla \boldsymbol{u}|\right)+(\alpha-2)\left(|\boldsymbol{w}|,|\boldsymbol{u}|^{\alpha-2}|\nabla \boldsymbol{u}|\right) \\
& \leq(\alpha-1)\left(|\boldsymbol{w}|,\left.\boldsymbol{u}\right|^{\alpha-2}|\nabla \boldsymbol{u}|\right) .
\end{aligned}
$$

Since $\frac{1}{\alpha}+\frac{\alpha-2}{2 \alpha}+\frac{1}{2}=1$, from (22) together the Hölder and Young inequalities, we get

$$
\begin{aligned}
2 \mu_{r}\left(\boldsymbol{w}, \operatorname{rot}\left(|\boldsymbol{u}|^{\alpha-2} \boldsymbol{u}\right)\right) & \leq 2 \mu_{r}(\alpha-1)\|\boldsymbol{w}\|_{\alpha}\left\||\boldsymbol{u}|^{\frac{\alpha}{2}-1}\right\|_{\frac{2 \alpha}{\alpha-2}}\left\||\boldsymbol{u}|^{\frac{\alpha}{2}-1}|\nabla \boldsymbol{u}|\right\|_{2} \\
& \leq 2 v_{1}(\alpha-1)\|\boldsymbol{w}\|_{\alpha}\||\boldsymbol{u}|\|_{\alpha}^{\frac{\alpha-2}{2}}\left(N_{\alpha}^{\alpha}(\boldsymbol{u})\right)^{1 / 2} \\
& \leq 4 v_{1}(\alpha-1)^{2}\|\boldsymbol{w}\|_{\alpha}^{2}\||\boldsymbol{u}|\|_{\alpha}^{\alpha-2}+\frac{v_{1}}{4} N_{\alpha}^{\alpha}(\boldsymbol{u}) .
\end{aligned}
$$


Again, since $\frac{2}{\alpha}+\frac{\alpha-2}{\alpha}=1$, by using the Young's inequality in (23), we obtain

$$
2 \mu_{r}\left(\boldsymbol{w}, \operatorname{rot}\left(|\boldsymbol{u}|^{\alpha-2} \boldsymbol{u}\right)\right) \leq C\left(\alpha, v_{1}\right)\|\boldsymbol{w}\|_{\alpha}^{\alpha}+C(\alpha)\|\boldsymbol{u}\|_{\alpha}^{\alpha}+\frac{v_{1}}{4} N_{\alpha}^{\alpha}(\boldsymbol{u}),
$$

Similarly as to (23) and (24), we can obtain

$$
\begin{aligned}
2 \mu_{r}\left(\boldsymbol{u}, \operatorname{rot}\left(|\boldsymbol{w}|^{\alpha-2} \boldsymbol{w}\right)\right) & \leq \frac{2 \mu_{r}^{2}(\alpha-1)^{2}}{v_{2}}\|\boldsymbol{u}\|_{\alpha}^{2}\||\boldsymbol{w}|\|_{\alpha}^{\alpha-2}+\frac{v_{2}}{2} N_{\alpha}^{\alpha}(\boldsymbol{w}) \\
& \leq C\left(\alpha, \mu_{r}\right)\|\boldsymbol{u}\|_{\alpha}^{\alpha}+C\left(\alpha, v_{2}\right)\|\boldsymbol{w}\|_{\alpha}^{\alpha}+\frac{v_{2}}{2} N_{\alpha}^{\alpha}(\boldsymbol{w})
\end{aligned}
$$

Now, considering (13) and since $\frac{1}{\alpha}+\frac{\alpha-1}{\alpha}=1$, by using the Young's inequality in (23), we have

$$
\left|\left(\boldsymbol{f},|\boldsymbol{u}|^{\alpha-2} \boldsymbol{u}\right)\right| \leq\|\boldsymbol{f}\|_{\alpha}\||\boldsymbol{u}|\|_{\alpha}^{\alpha-1} \leq C\|\boldsymbol{f}\|_{\alpha}^{\alpha}+C\||\boldsymbol{u}|\|_{\alpha}^{\alpha} .
$$

Similarly

$$
\left|\left(\boldsymbol{g},|\boldsymbol{w}|^{\alpha-2} \boldsymbol{w}\right)\right| \leq C\|\boldsymbol{g}\|_{\alpha}^{\alpha}+C\||\boldsymbol{w}|\|_{\alpha}^{\alpha}
$$

Now, taking into account that $\left.\left.|\nabla| \boldsymbol{u}\right|^{\frac{\alpha}{2}}\left|=\frac{\alpha}{2}\right| \boldsymbol{u}\right|^{\frac{\alpha}{2}-1}|\nabla| \boldsymbol{u}||$ a.e. in $\Omega$, to the last term in the right-hand side of (21), we get

$$
\begin{aligned}
\left(\operatorname{div} \boldsymbol{w},|\boldsymbol{w}|^{\alpha-3} \boldsymbol{w} \cdot \nabla|\boldsymbol{w}|\right) & \leq\left(|\operatorname{div} \boldsymbol{w}|,|\boldsymbol{w}|^{\alpha-2}|\nabla| \boldsymbol{w} \|\right) \\
& \leq\left(|\operatorname{div} \boldsymbol{w}||\boldsymbol{w}|^{\frac{\alpha}{2}-1},|\boldsymbol{w}|^{\frac{\alpha}{2}-1}|\nabla| \boldsymbol{w} \|\right) \\
& \leq\left\||\operatorname{div} \boldsymbol{w}||\boldsymbol{w}|^{\frac{\alpha}{2}-1}\right\|_{2}\left\||\boldsymbol{w}|^{\frac{\alpha}{2}-1}|\nabla| \boldsymbol{w}\right\|_{2} \\
& \leq \frac{2}{\alpha}\left\||\operatorname{div} \boldsymbol{w}||\boldsymbol{w}|^{\frac{\alpha}{2}-1}\right\|_{2}\left\|\left.|\nabla| \boldsymbol{w}\right|^{\frac{\alpha}{2}} \mid\right\|_{2} .
\end{aligned}
$$

Then, by using the Young's inequality, from (28) we have

$$
\left(\operatorname{div} \boldsymbol{w},|\boldsymbol{w}|^{\alpha-3} \boldsymbol{w} \nabla|\boldsymbol{w}|\right) \leq \frac{1}{\alpha}\left\||\boldsymbol{w}|^{\frac{\alpha}{2}-1}|\operatorname{div} \boldsymbol{w}|\right\|_{2}^{2}+\frac{1}{\alpha} M_{\alpha}^{\alpha}(\boldsymbol{w})
$$

and since, $\frac{1}{3}<\frac{\alpha-2}{\alpha}<1$ for $\alpha>3$, then

(29) $v_{3}(\alpha-2)\left(\operatorname{div} \boldsymbol{w},|\boldsymbol{w}|^{\alpha-3} \boldsymbol{w} \nabla|\boldsymbol{w}|\right) \leq v_{3}\left\||\boldsymbol{w}|^{\frac{\alpha}{2}-1}|\operatorname{div} \boldsymbol{w}|\right\|_{2}^{2}+v_{3} \frac{(\alpha-2)}{\alpha} M_{\alpha}^{\alpha}(\boldsymbol{w})$.

Setting (24) and(26) in (20), as well as (25), (27) and (29) in (21), we obtain

$$
\begin{aligned}
& \frac{1}{\alpha} \frac{d}{d t}\|\boldsymbol{u}\|_{\alpha}^{\alpha}+\frac{3 v_{1}}{4} N_{\alpha}^{\alpha}(\boldsymbol{u})+4 v_{1} \frac{(\alpha-2)}{\alpha^{2}} M_{\alpha}^{\alpha}(\boldsymbol{u}) \\
& \leq-\left(\nabla p,|\boldsymbol{u}|^{\alpha-2} \boldsymbol{u}\right)+C\|\boldsymbol{w}\|_{\alpha}^{\alpha}+C\|\boldsymbol{u}\|_{\alpha}^{\alpha}+C\|\boldsymbol{f}\|_{\alpha}^{\alpha} .
\end{aligned}
$$


and

$$
\begin{aligned}
& \frac{1}{\alpha} \frac{d}{d t}\|\boldsymbol{w}\|_{\alpha}^{\alpha}+\frac{v_{2}}{2} N_{\alpha}^{\alpha}(\boldsymbol{w})+\left(4 v_{2}-\alpha v_{3}\right) \frac{(\alpha-2)}{\alpha^{2}} M_{\alpha}^{\alpha}(\boldsymbol{w})+4 \mu_{r}\|\boldsymbol{w}\|_{\alpha}^{\alpha} \\
& \leq C\|\boldsymbol{u}\|_{\alpha}^{\alpha}+C\|\boldsymbol{w}\|_{\alpha}^{\alpha}+C\|\boldsymbol{g}\|_{\alpha}^{\alpha},
\end{aligned}
$$

thus adding inequalities (30) and (31) is followed (19).

LEMMA 3.2. Let $(\boldsymbol{u}, p, \boldsymbol{w})$ be a smooth solution of the micropolar equations (1)-(3) in $\Omega \times(0, T]$. Then the following estimate is satisfied

$$
-\left(\nabla p,|\boldsymbol{u}|^{\alpha-2} \boldsymbol{u}\right) \leq C_{2}\|p\|_{\frac{3 \alpha}{\alpha+1}}^{\alpha}+v_{1} \frac{(\alpha-2)}{\alpha^{2}} M_{\alpha}^{\alpha}(\boldsymbol{u})+\frac{v_{1}}{4} N_{\alpha}^{\alpha}(\boldsymbol{u}),
$$

where $C_{2}$ is a positive constant dependent on $v_{1}, \alpha$.

Proof. Taking into account that $\boldsymbol{u} \in H_{\alpha}$ and (12), we have

$$
\begin{aligned}
-\left(\nabla p,|\boldsymbol{u}|^{\alpha-2} \boldsymbol{u}\right) & =\left(p, \operatorname{div}\left(|\boldsymbol{u}|^{\alpha-2} \boldsymbol{u}\right)\right)=(\alpha-2)\left(p,|\boldsymbol{u}|^{\alpha-3} \boldsymbol{u} \cdot \nabla|\boldsymbol{u}|\right) \\
& \leq(\alpha-2)\left(|p|,|\boldsymbol{u}|^{\alpha-2}|\nabla \boldsymbol{u}|\right) \\
& \leq(\alpha-2)\left(|p||\boldsymbol{u}|^{\frac{\alpha}{2}-1},|\boldsymbol{u}|^{\frac{\alpha}{2}-1}|\nabla \boldsymbol{u}|\right) .
\end{aligned}
$$

Since $\frac{\alpha+1}{3 \alpha}+\frac{\alpha-2}{6 \alpha}+\frac{1}{2}=1$, by applying Hölder's inequality in (33), we have

$$
\begin{aligned}
-\left(\nabla p,|\boldsymbol{u}|^{\alpha-2} \boldsymbol{u}\right) & \leq(\alpha-2)\|p\|_{\frac{3 \alpha}{\alpha+1}}\left\||\boldsymbol{u}|^{\frac{\alpha}{2}-1}\right\|\left\|_{\frac{6 \alpha}{\alpha-2}}\right\||\boldsymbol{u}|^{\frac{\alpha}{2}-1}|\nabla \boldsymbol{u}| \|_{2} \\
& \leq(\alpha-2)\|p\|_{\frac{3 \alpha}{\alpha+1}}\|\boldsymbol{u}\|_{3 \alpha}^{\frac{\alpha-2}{2}}\left\||\boldsymbol{u}|^{\frac{\alpha}{2}-1}|\nabla \boldsymbol{u}|\right\|_{2}
\end{aligned}
$$

Then, by using the Young's inequality in (34), we get

$$
-\left(\nabla p,|\boldsymbol{u}|^{\alpha-2} \boldsymbol{u}\right) \leq \frac{(\alpha-2)^{2}}{v_{1}}\|p\|_{\frac{3 \alpha}{\alpha+1}}^{2}\|\boldsymbol{u}\|_{3 \alpha}^{\alpha-2}+\frac{v_{1}}{4} N_{\alpha}^{\alpha}(\boldsymbol{u}) .
$$

Now, $\frac{2}{\alpha}+\frac{\alpha-2}{\alpha}=1$, then by using the Young's inequality and (8), we have

$$
\begin{aligned}
\frac{(\alpha-2)^{2}}{v_{1}}\|p\|_{\frac{3 \alpha}{\alpha+1}}^{2}\|\boldsymbol{u}\|_{3 \alpha}^{\alpha-2} & \leq C_{2}\|p\|_{\frac{3 \alpha}{\alpha+1}}^{\alpha}+v_{1} \frac{(\alpha-2)}{\alpha^{2}}\left(\frac{1}{C_{0}}\right)\|\boldsymbol{u}\|_{3 \alpha}^{\alpha} \\
& \leq C_{2}\|p\|_{\frac{3 \alpha}{\alpha+1}}^{\alpha}+v_{1} \frac{(\alpha-2)}{\alpha^{2}} M_{\alpha}^{\alpha}(\boldsymbol{u}),
\end{aligned}
$$

where $C_{2}=\left(\frac{\alpha-2}{v_{1}}\right)^{\alpha}\left(C_{0} \alpha\right)^{\frac{\alpha-2}{2}}\left(\frac{2 v_{1}}{\alpha}\right)$.

Thus, inequalities (35) and (36) imply (32). 


\section{Proof of Theorem 2.1}

The proof of Theorem 2.1 is based on Lemma 3.1 and Lemma 3.2 with the aid of smoothing properties.

From Lemma 3.1 and Lemma 3.2, we have that the smooth solution $(\boldsymbol{u}, p, \boldsymbol{w})$ of the micropolar equations (1)-(3) satisfies

$$
\begin{aligned}
& \frac{d}{d t}\left(\|\boldsymbol{u}\|_{\alpha}^{\alpha}+\|\boldsymbol{w}\|_{\alpha}^{\alpha}\right)+\frac{\alpha v_{1}}{2} N_{\alpha}^{\alpha}(\boldsymbol{u})+\frac{\alpha v_{2}}{2} N_{\alpha}^{\alpha}(\boldsymbol{w})+3 v_{1} \frac{(\alpha-2)}{\alpha} M_{\alpha}^{\alpha}(\boldsymbol{u}) \\
& \quad+C_{1} \frac{(\alpha-2)}{\alpha} M_{\alpha}^{\alpha}(\boldsymbol{w}) \leq C_{2}\|p\|_{\frac{3 \alpha}{\alpha+1}}^{\alpha}+C\left(\|\boldsymbol{f}\|_{\alpha}^{\alpha}+\|\boldsymbol{g}\|_{\alpha}^{\alpha}\right)+C\left(\|\boldsymbol{u}\|_{\alpha}^{\alpha}+\|\boldsymbol{w}\|_{\alpha}^{\alpha}\right) .
\end{aligned}
$$

Moreover, by observing the notation given in (7) and since $\frac{\alpha}{2}>1$, $\frac{\alpha-2}{\alpha}>\frac{1}{3}$, from (37) we get

$$
\begin{gathered}
\frac{d}{d t}\left(\|\boldsymbol{u}\|_{\alpha}^{\alpha}+\|\boldsymbol{w}\|_{\alpha}^{\alpha}\right)+v_{1} N_{\alpha}^{\alpha}(\boldsymbol{u})+v_{2} N_{\alpha}^{\alpha}(\boldsymbol{w})+v_{1}\left\|\nabla|\boldsymbol{u}|^{\frac{\alpha}{2}}\right\|_{2}^{2}+\frac{C_{1}}{3}\left\|\nabla|\boldsymbol{w}|^{\frac{\alpha}{2}}\right\|_{2}^{2} \\
\leq C_{2}\|p\|_{\frac{3 \alpha}{\alpha+1}}^{\alpha}+C\left(\|\boldsymbol{f}\|_{\alpha}^{\alpha}+\|\boldsymbol{g}\|_{\alpha}^{\alpha}\right)+C\left(\|\boldsymbol{u}\|_{\alpha}^{\alpha}+\|\boldsymbol{w}\|_{\alpha}^{\alpha}\right) .
\end{gathered}
$$

By integrating (38) from 0 to $t \in(0, T)$, we have

$$
\begin{aligned}
& \|\boldsymbol{u}(t)\|_{\alpha}^{\alpha}+\|\boldsymbol{w}(t)\|_{\alpha}^{\alpha}+v_{1} \int_{0}^{t} N_{\alpha}^{\alpha}(\boldsymbol{u}(\tau)) d \tau+v_{2} \int_{0}^{t} N_{\alpha}^{\alpha}(\boldsymbol{w}(\tau)) d \tau \\
& +v_{1} \int_{0}^{t}\left\|\nabla|\boldsymbol{u}(\tau)|^{\frac{\alpha}{2}}\right\|_{2}^{2} d \tau+\frac{C_{1}}{3} \int_{0}^{t}\left\|\nabla|\boldsymbol{w}(\tau)|^{\frac{\alpha}{\alpha}}\right\|_{2}^{2} d \tau \\
& \leq\left\|\boldsymbol{u}_{0}\right\|_{\alpha}^{\alpha}+\left\|\boldsymbol{w}_{0}\right\|_{\alpha}^{\alpha}+C \int_{0}^{t}\|p(\tau)\|_{\frac{3 \alpha}{\alpha+1}}^{\alpha} d \tau+C \int_{0}^{t}\left(\|\boldsymbol{f}(\tau)\|_{\alpha}^{\alpha}+\|\boldsymbol{g}(\tau)\|_{\alpha}^{\alpha}\right) d \tau \\
& +C \int_{0}^{t}\left(\|\boldsymbol{u}(\tau)\|_{\alpha}^{\alpha}+\|\boldsymbol{w}(\tau)\|_{\alpha}^{\alpha}\right) d \tau .
\end{aligned}
$$

Then, taking into account the hypotheses on $\boldsymbol{f}, \boldsymbol{g}$ and $p$ (see (14)), inequality (39) implies

$$
\|\boldsymbol{u}(t)\|_{\alpha}^{\alpha}+\|\boldsymbol{w}(t)\|_{\alpha}^{\alpha}+v_{1} \int_{0}^{t} N_{\alpha}^{\alpha}(\boldsymbol{u}(\tau)) d \tau+v_{2} \int_{0}^{t} N_{\alpha}^{\alpha}(\boldsymbol{w}(\tau)) d \tau+v_{1} \int_{0}^{t}\left\|\nabla|\boldsymbol{u}(\tau)|^{\frac{\alpha}{2}}\right\|^{2} d \tau
$$

$$
+\frac{C_{1}}{3} \int_{0}^{t}\left\|\nabla|\boldsymbol{w}(\tau)|^{\frac{\alpha}{2}}\right\|^{2} d \tau \leq C+C \int_{0}^{t}\left(\|\boldsymbol{u}(\tau)\|_{\alpha}^{\alpha}+\|\boldsymbol{w}(\tau)\|_{\alpha}^{\alpha}\right) d \tau .
$$


and using the Gronwall's inequality in (40), we obtain

$$
\begin{gathered}
\|\boldsymbol{u}(t)\|_{\alpha}^{\alpha}+\|\boldsymbol{w}(t)\|_{\alpha}^{\alpha}+v_{1} \int_{0}^{t}\left\|\nabla|\boldsymbol{u}(\tau)|^{\frac{\alpha}{2}}\right\|^{2} d \tau+\frac{C_{1}}{3} \int_{0}^{t}\left\|\nabla|\boldsymbol{w}(\tau)|^{\frac{\alpha}{2}}\right\|^{2} d \tau \\
\leq C(1+t) \exp (C t):=K(t) \leq K(T)
\end{gathered}
$$

then follows that $(\boldsymbol{u}, \boldsymbol{w}) \in L^{\infty}\left(0, t ; H_{\alpha}\right) \times L^{\infty}\left(0, t ; G_{\alpha}\right)$ and consequently by the hypothesis $\alpha>3$ (see [8]) we have that $(\boldsymbol{u}, \boldsymbol{w}) \in C\left(0, t ; H_{\alpha}\right) \times C\left(0, t ; G_{\alpha}\right)$.

Now, if we denote by $t^{*}$ the lowest upper bound of the values in $[0, T]$ for which (41) is satisfied in $\left[0, t^{*}\right]$, we have that $t^{*}>0$ and $(\boldsymbol{u}, \boldsymbol{w}) \in C\left(0, t^{*} ; H_{\alpha}\right) \times$ $\times C\left(0, t^{*} ; G_{\alpha}\right)$. Then, taking $\boldsymbol{u}\left(t^{*}\right), \boldsymbol{w}\left(t^{*}\right)$ as initial data, if $t^{*}<T$ one show from (39) and (41) that exists a positive constant $\eta$ such that

$$
\begin{gathered}
\left\|\boldsymbol{u}\left(t^{*}+\eta\right)\right\|_{\alpha}^{\alpha}+\left\|\boldsymbol{w}\left(t^{*}+\eta\right)\right\|_{\alpha}^{\alpha}+v_{1} \int_{t^{*}}^{t^{*}+\eta}\left\|\nabla|\boldsymbol{u}(\tau)|^{\frac{\alpha}{2}}\right\|^{2} d \tau \\
+\frac{C_{1}}{3} \int_{t^{*}}^{t^{*}+\eta}\left\|\nabla|\boldsymbol{w}(\tau)|^{\frac{\alpha}{2}}\right\|^{2} d \tau \leq K(\eta) \leq K(T),
\end{gathered}
$$

thus $(\boldsymbol{u}, \boldsymbol{w}) \in C\left(0, t^{*}+\eta ; H_{\alpha}\right) \times C\left(0, t^{*}+\eta ; G_{\alpha}\right)$, therefore necessarily it must be $t^{*}=T$.

REMARK 4.1. For $\alpha>3$ we have that $\frac{9}{4}<\frac{3 \alpha}{\alpha+1}<3$, then

$$
L^{\infty}\left(0, T ; L^{q}(\Omega)\right) \subset L^{\alpha}\left(0, T ; L^{\frac{3 \alpha}{\alpha+1}}(\Omega)\right), \quad \frac{9}{4}<q<3,
$$

where $q=\frac{3 \alpha}{\alpha+1}$. Thus, from (14) and (43), we conclude that the same result of the Theorem 2.1 is obtained if we consider

$$
p \in L^{\infty}\left(0, T ; L^{q}(\Omega)\right), \quad \frac{9}{4}<q<3 .
$$

Moreover, since $\frac{12}{5}>\frac{9}{4}$ the condition on the pressure given by Kaniel in [10] is improved.

\section{REFERENCES}

[1] H. Beirão DA VEIGA, A new regularity class for the Navier-Stokes equations in $\mathbb{R}^{n}$, Chin. Ann. of Math., 16B, 4 (1995), pp. 1-6.

[2] H. BEIRÃo DA VEIGA, Concerning the regularity of the solutions to the NavierStokes equations via the truncation method Part II, In Equations aux dérivées partielles et applications Gauthier-Villars Éd. Sci. Méd. Elsevier (Paris 1998), pp. 127-138.

[3] H. Beirão da Veiga, A Sufficient condition on the pressure for the regularity 
of weak Solutions to the Navier-Stokes equations, J. Math. Fluid Mech., 2 (2000), pp. 99-106.

[4] H. BEIRÃO DA VEIGA, Existence and asymptotic behavior for strong solutions of the Navier-Stokes equations in the whole space, Indiana Univ. Math. J., 36 (1987), pp. 149-166.

[5] L. BERSELLI, Sufficient conditions for the regularity of the solutions of the Navier-Stokes Equations, Math. Meth. Appl. Sci., 22 (1999), pp. 1079-1085.

[6] D. CHAE - J. LEE, Regularity criterion in terms of pressure for the Navier-Stokes equations, Nonlinear Anal., 46, no. 5, Ser. A: Theory Methods (2001), pp. 727-735.

[7] A. C. ERIngen, Theory of micropolar fluids, J. Math. Mech., 16 (1966), pp. 1-8.

[8] Y. GIYA - T. MiYakawa, Solutions in $L^{r}$ to the Navier-Stokes initial value problem, Arch. Rat. Mech. Anal., 89 (1985), pp. 267-281.

[9] J. G. HEYWOOD - O. D. WALSH, A counter-example concerning the pressure in the Navier-Stokes, as $t \rightarrow 0^{+}$, Pacific J. Math., 164 (1994), pp. 351-359.

[10] S. KANIEL, A sufficient conditions for smoothness of solutions of NavierStokes equations, Israel J. Math., 6 (1968), pp. 354-358.

[11] S. KaniEL - M. Shinbrot, Smoothness of weak solutions of the Navier-Stokes equations, Arch. Rational Mech. Anal., 24 (1967), pp. 302-324.

[12] O. A. Ladyzhenskaya, The mathematical theory of viscous incompressible flow, Second edition, Gordon and Breach (New York 1969).

[13] G. LuKaszewICZ, On the existence, uniqueness and asymptotic properties of solutions of flows of asymmetric fluids, Rend. Accad. Naz. Sci. XL, Mem. Math., 107 (vol. XIII) (1989), pp. 105-120.

[14] G. Lukaszewicz, Micropolar fluids: theory and applications, Birkhäuser (Berlin 1998).

[15] K. MASUdA, Weak solutions of Navier-Stokes equations, Tohoku Math. J., 36 (1984), pp. 623-646.

[16] M. O'LERAY, Pressure conditions for the local regularity of solutions of the Navier-Stokes equations, EJDE, 1998 (1998), pp. 1-9.

[17] E. Ortega-Torres - M. A. Rojas-Medar, On the uniqueness and regularity of the weak solutions for magneto-micropolar equations, Rev. Mat. Apl., 17 (1996), pp. 75-90.

[18] M. A. RoJAs-Medar - J. L. BoldRInI, Magneto-micropolar fluid motion: existence of weak solution, Rev. Mat. Univ. Complutense de Madrid., Vol. 11, 2 (1998), pp. 443-460.

[19] J. SERRIN, On the interior regularity of weak solutions of the Navier-Stokes equations, Arch. Rat. Mech. Anal., 9 (1962), pp. 187-195.

[20] H. SoHR, The Navier-Stokes equations, a elementary functional analytic approach, Birkhäuser (Berlin 2001).

[21] R. Temam, Navier-Stokes equations, theory and numerical analysis, North Holland ( ${ }^{\text {nd }}$ Revised Edition) (Amsterdam 1979).

[22] W. Von WhalH, Regularity question for the Navier-Stokes equations, in: R. Rautmann ed., Approximations Methods for the Navier-Stokes Problems, Lectures and Notes in Mathematics, 771 (Springer-Verlag, Berlin 1980), pp. 538-542.

[23] N. YAMAGUCHI, Existence of global solution to the micropolar fluid system in a bounded domain, Math. Method Appl. Sci., 28 (2005), pp. 1507-1526.

Manoscritto pervenuto in redazione il 6 marzo 2008. 
